\title{
Enhanced Recovery After Surgery for Low- and Middle-Income Countries
}

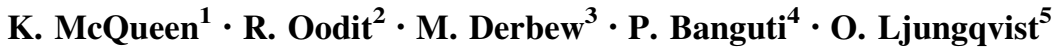

Published online: 30 January 2018

(C) Société Internationale de Chirurgie 2018

The revolution of Enhanced Recovery After Surgery (ERAS) is sweeping high-income countries (HICs), improving surgical and anesthesia complication rates and outcomes, and saving healthcare dollars due to fewer complications and decreased length of stay in the hospital [1]. But ERAS is not only for HICs. We propose that a modified approach to ERAS would extend protocols to improving all types of surgery in low- and lower-middleincome countries (LMICs) and would proactively create responsible anesthesia, perioperative, and surgical systems that are cost and technology appropriate, and limit complications and hospital stays.

Surgical services have long been neglected in low- and middle-income countries (LMICs) primarily due to the prevalence of communicable disease as the largest contributor to disability and death. Many related myths contributed to limited support for the development of surgical and anesthesia services in LMICs including assumptions such as "surgery is too expensive" or "surgical disease is too complicated." In fact, surgery and anesthesia have been shown to be cost-effective [2], to support generalized improvement of hospital and healthcare infrastructure, and that without addressing the burden of surgical disease, significant GDP will be lost for most LMICs [3]. The shift

\footnotetext{
K. McQueen

kamcqueen@gmail.com

1 Department of Anesthesiology, Vanderbilt University, Nashville, South Africa

2 University of Cape Town, Cape Town, South Africa

3 Black Lion Hospital, Addis Ababa, Ethiopia

4 Department of Anesthesiology, College of Medicine and Health Sciences, University of Rwanda, Kigali, Rwanda

5 Orebro Universitet, Örebro, Sweden
}

in global disease burdens away from communicable disease and toward non-communicable disease (NCDs) demand the growth of surgical services. Non-communicable diseases, especially trauma and cancer, frequently require surgery and anesthesia for diagnosis, treatment, and early resuscitation.

The events of 2015 [2, 3] culminating the unanimously passed World Health Assembly Resolution 68.15 [4] have elevated the discussions on inclusion of surgery and anesthesia in global health and national healthcare plans. Led by efforts at the World Health Organization (WHO), the scale up to 44 basic surgeries and safe anesthesia is underway, and in fact two Africa countries have proposed national surgical plans for the process $[5,6]$. As proposed by the Lancet Commission on Global Surgery and the WHO, this is an opportunity for tracking and improving outcomes, and initiating quality programs early in the scale up processes. It is timely to consider the benefits that ERAS protocols offer and to propose the standardization and audit system that will prevent complications, save lives and save healthcare dollars. The ERAS concepts for LMICs have already been proposed at two internal meetings.

\section{ERAS in High-Income Countries}

Surgery in HICs has been long supported as an essential healthcare component, and advances in medicine and technology lead to escalating trajectory of surgical delivery since the 1950s. (REF History). These advances prevented premature disability and from common surgical conditions such as appendicitis, common and uncommon cancers, and complicated traumatic injuries alike. Over decades, technological advances in surgery and anesthesia allowed for the care of more complicated and sicker patients, and 
seemingly there were no limits new technology and its application, nor the patient population that benefited from the interventions. By the 1990s, however, it became apparent that the escalating costs associated with these advances could not continue unabated.

Enhancing recovery using a multimodal approach was first conceptualized by Henrik Kehlet, a Danish surgeon in the 1990s [1] as Fast Track surgery. He also proved its efficacy with revolutionary results when employing it to colonic resections with patients ready to discharge in 2 days instead of 2 weeks. These ideas were further developed by a larger group publishing an evidence-based guidance to perioperative care in the following decade [4], and also showed that it is possible to help other units to implement the guideline protocol in less than a year and reduce recovery time by a third. This group coined the term Enhanced Recovery After Surgery (ERAS) placing the focus on the patient's recovery, including improving complication rates and hospital discharge times. This group later formed a nonprofit medical society-the ERAS $^{\circledR}$ Society (www.erassociety.org), which is now a network of committed individuals, societies, hospitals and academic centers.

The spread of these concepts is growing with the increase in data that are published to show the effectiveness of ERAS principles. With the introduction of ERAS, many specific disease pathways have made significant impact on the patient outcomes by reducing complications $40-50 \%$ for major operations [7], while at the same time decreasing hospital length of stay and therefore costs [8] For example, in Alberta, Canada, where the state has instituted a statewide ERAS implementation program, the return in the investment made on the program is around four times the cost of the investment [9].

\section{ERAS for LMICs}

The situation in many LMICs, especially the lowest income countries, is comparable to the state of surgery in HICs as many as five decades ago. This reality is an incredible opportunity for the standardization of care as scale up progresses, and for impacting outcomes, complications, and length of stay in a proactive manner. The potential long-term health and cost savings will be impactful [10], and has the potential to avoid many HIC mistakes (narcotic dependence, pain syndromes, complications related to long hospital stays, costs.)

A discussion on ERAS and its application in LMICs is prudent as National Surgery Plans have been proposed by LCoGS as the next step for LMICs as scale up of surgery and anesthesia. Designing the surgical and anesthesia landscape with the ERAS principles and protocols in mind will lead to cost-effective approaches as well as a quality- centric system that focuses on preventing complications, improving outcomes, and limiting hospital stays. If National Surgery plans were to include the essential pathways for optimal surgical care: preoperative evaluation, intraoperative standardized care, and postoperative management including early ambulation and multimodal pain management, it is likely that surgical systems would grow optimally, with fewer complications and better outcomes.

The global focus on surgery and anesthesia during the 15-year span 2015-30 is a unique opportunity to consider the optimal scale up to surgical and anesthesia protocols and processes, and to build-in often neglected aspects of surgical care such as preoperative evaluation and optimization, intraoperative standardization, and follow-up programs. Additional important components of an effective surgical system include availability of essential medicines [3], training for anesthesia providers in regional anesthesia techniques, avoiding the use of many unnecessary treatments such as drains and tubes and too much intravenous fluids, and the use of the increased availability of modern IT technology and data management also in the LMIC for control of the care processes and outcomes.

Surgery and anesthesia outcomes would be benefited by anesthesia and surgery protocols, such as those proposed by many ERAS programs. In LMICs these protocols would ideally focus on preoperative evaluation and optimization, available, cost-effective medications including antibiotics, regional blockade, multimodal pain management, early mobilization postoperatively. Implementation of modified ERAS protocols for all 44 surgical procedures currently recommended as cost-effective with anesthesia for all first referral hospitals in LMICs has the potential to improve the surgical pathway processes, create preoperative and postoperative plans and standards, limit costs, and improve outcomes for all patients.

\section{References}

1. Ljungqvist O, Scott M, Fearon KC (2017) Enhanced recovery after surgery: a review. JAMA Surg 152(3):292-298

2. Debas HT, Donkor P, Gawande A, Jamison DT, Kruk ME, Mock $\mathrm{CN}$, eds. Essential surgery. Disease control priorities. Vol 1. 3rd ed. Washington, DC: The International Bank for Reconstruction and Development/The World Bank; 2015. Available at: http:// documents.worldbank.org/curated/en/655391468130824512/ Essential-surgery. Accessed 23 Nov 2016

3. Meara JG, Leather AJ, Hagander L et al (2015) Global surgery 2030: evidence and solutions for achieving health, welfare, and eco-nomic development. Lancet 386:569-624

4. WHA Resolution 68.15 (2015) Strengthening emergency and essential surgical care and anaesthesia as a component of universal health coverage. World Health Assembly, Geneva. Available at: http://apps.who.int/medicinedocs/documents/ s21904en/s21904en.pdf. Accessed 29 Oct 2017 
5. Mukhopadhyay S, Lin Y, Mwaba P et al (2017) Implementing World Health Assembly Resoltion 68.15: national surgical, obstetric and anesthesia strategic plan development-the Zambian experience. Bull Am Coll Surg 102(6):28-35

6. Burssa D, Teshome A, Iverson K et al (2017) Safe surgery for all: early lessons from implementing a national government-driven surgical plan in Ethiopia. World J Surg 41(12):3038-3045. https://doi.org/10.1007/s00268-017-4271-5

7. Fearon $\mathrm{KCH}$, Ljungqvist $\mathrm{O}$, Von Meyenfeldt M, Nygren J, Hausel J, Soop M (2005) Enhanced recovery after surgery: a consensus review of clinical care for patients undergoing colonic resection. Clin Nutr 24:466-477
8. Greco M, Capretti G, Beretta L, Gemma M, Pecorelli N, Braga M (2014) Enhanced recovery program in colorectal surgery: a metaanalysis of randomized controlled trials. World J Surg 38(6):1531-1541

9. Ljungqvist O, Nguyen XT, Nelson G (2017) ERAS—value based surgery. J Surg Oncol 116(5):608-612

10. Thanh NX, Chuck AW, Wasylak T, Lawrence J, Faris P, Ljungqvist O, Nelson G, Gramlich LM (2016) An economic evaluation of the Enhanced Recovery After Surgery (ERAS) multisite implementation program for colorectal surgery in Alberta. Can J Surg 59(6):6716 